\title{
2D to 3D Evaluation of Organs at Risk Doses in Intracavitary Brachytherapy for Cervical Cancer
}

\author{
Yun Inn Tan, MSc, Bok Ai Choo, MBChB, MRCP, FRCR, Khai Mun Lee, MBBS, FFRRCSI, FRCR, FAMS \\ Lower Kent Ridge Road Radiotherapy Centre, National University Cancer Institute, Singapore
}

\begin{abstract}
Purpose: To compare International Commission on Radiation Units and Measurements (ICRU) bladder and rectum reference points doses with volumetric doses in 3D intracavitary brachytherapy (ICBT) for cervical cancer. Also to compare bladder, rectum and sigmoid (organs at risk, OARs) volume doses with dose constraints recommended by the (GYN) GEC-ESTRO Working Group.

Material and methods: A retrospective study was carried out on 10 patients with a total of 55 fractions CT-based high dose rate (HDR) ICBT. ICRU bladder (bICRU) and rectum (rICRU) points were defined according to ICRU Report 38 on the CT images and prospectively kept to less than $80 \%$ of prescription dose to Point A during real treatment planning. Post-treatment, outer wall of OARs were contoured and minimum dose to 2cc (D2cc) of the most irradiated part of the OARs was obtained from the dose-volume histogram (DVH). Total dose (external beam radiotherapy plus ICBT) were computed with ICRU point dose and D2cc and compared.

Results: The mean ICRU point dose and D2cc volume dose were found to be significantly different for bladder (per fraction: $p=0.000$; total dose: $p=0.004$ ) but no differences were found for rectum (per fraction: $p=0.055$; total dose: $p=0.090$ ). bICRU point dose underestimated D2cc dose with an average ratio of $1.34 \pm 0.34 .3$ out of 10 patients, 7 out of 10 patients, and 5 out of 10 patients exceeded the recommended dose constraint for bladder, rectum, and sigmoid, respectively.

Conclusions: bICRU was not representative of bladder D2cc and resulted in different total dose. rICRU was found to be similar to D2cc dose and was reliable in total dose computation. Our current institutional practice of point-based planning in ICBT resulted in significant number of patients' OARs doses exceeded the volume constraint, because the total dose concept was not used propectively in planning.
\end{abstract}

J Contemp Brachyther 2010; 2, 1: 37-43

Key words: brachytherapy, cervical cancer, CT-based, intracavitary.

\section{Purpose}

Traditionally, bladder and rectum dose in intracavitary brachytherapy (ICBT) for cervical cancer are estimated using the International Commission on Radiation Units and Measurements (ICRU) reference points [1]. The ICRU points were an acceptable way of estimating dose to bladder and rectum only if 2D orthogonal films were available for ICBT planning. However, the correlation of the ICRU point doses with bladder and rectal complications are debatable [2-5]. With the availability of CT scanner and treatment planning software for 3D brachytherapy in recent years, there is a need to move forward to a $3 \mathrm{D}$ assessment of organs at risk (OARs). Instead of using point doses, volumetric assessment of OARs is a better and more complete representation of doses to OARs [6-8].

3D brachytherapy planning became available in National University Cancer Institute Singapore (NCIS) in April 2008. Since then, all treatment plans for cervical cancer brachytherapy patients are designed using CT images for each application. Manchester System Point A prescription is still the standard prescription point and ICRU reference points for bladder and rectum are defined following the ICRU Report 38 [1]. Standard loading pattern is used to achieve the conventional pear-shaped distribution. No contour is drawn at the time of planning and total dose (dose from external beam radiotherapy, EBRT, plus ICBT) is not evaluated prospectively during planning.

As part of the transition from 2D to 3D planning, we hope to evaluate and achieve a better understanding of our current practice of point-based planning using CT images. In addition to the usual planning and treatment, post-treatment dosimetry assessments of OARs were being carried out. By doing so, ICRU bladder and rectum reference point doses can be compared with dose-volume histogram (DVH) doses. This study also aims to compare bladder, rectum and sigmoid doses with dose constraints recommended by the (GYN) GEC-ESTRO Working Group [9] to evaluate our current institutional protocol for ICBT.

Address for correspondence: Tan Yun Inn, MSc, National University Hospital System 5 Lower Kent Ridge Received: 03.03.10 Road Radiotherapy Centre, Level 3, Singapore 119074, Phone (65) 67724853, (65) 63571080, fax (65) 6779 6320, Accepted: 09.03 .10 (65) 6258 9236, e-mail: yun_inn_tan@nuhs.edu.sg 


\section{Material and methods}

\section{Patient selection}

Ten patients with a total of 55 fractions of CT-based high dose-rate (HDR) ICBT for cervical cancer were selected for this retrospective study. Selected patients were treated in NCIS between June 2008 and July 2009.

\section{Treatment scheme}

The standard treatment for cervical cancer consists of external beam radiotherapy (EBRT) using four-field box technique, with or without concomitant chemotherapy, and ICBT. In this study, EBRT dose was 45 Gy (2 patients) and 50.4 Gy (8 patients) delivered at 1.8 Gy per fraction. As for ICBT, prescription dose was 5.3 Gy to Point A in 5 fractions ( 5 patients) or 6 fractions ( 5 patients). ICBT was carried out on alternate days amounting to 2 to 3 treatments a week. We aim to complete the whole course of treatment within 50 days.

\section{Brachytherapy insertion and scanning}

Fletcher Williamson "Asia Pacific" metal ovoid applicators were used (ovoid sizes: half ovoid, $20 \mathrm{~mm}$, and 25 $\mathrm{mm}$; tandem angles: $15^{\circ}, 30^{\circ}$ and $45^{\circ}$ ). Combination of ovoid size and tandem angle were chosen according to patient's anatomy. Packing was done to set the applicators in place and also to displace the bladder and rectum. Foley balloon was inserted and filled with $7 \mathrm{cc}$ air and pulled to sit on the bladder trigone. All procedures were done under conscious sedation without general or spinal anesthetics. After that, patients were CT-scanned with applicators in place. Scanning was done with Philips Brilliance Big Bore CT scanner using $120 \mathrm{kV}, 325 \mathrm{mAs}$, and a combination of $3 \mathrm{~mm}$ and $5 \mathrm{~mm}$ slices. No contrast was used.

\section{Brachytherapy planning}

Planning were done using Oncentra Treatment Planning System (Nucletron). 5.3 Gy was prescribed to Point A of the Manchester System using standard loading pattern without optimization. Limited optimization was done in some patients whenever necessary. Mean TRAK value was $0.34 \pm 0.03$ cGy at $1 \mathrm{~m}$. Bladder and rectum points were defined according to ICRU Report 38 [1]. As a standard institutional practice, bladder and rectum point doses were kept to less than $80 \%$ of dose to Point A for each fraction, except in 3 applications where ICRU bladder point dose was exceeded. No contouring was done during the actual treatment planning.

\section{Post-treatment OARs delineation and treatment planning}

Post-treatment, radiation oncologists delineated the outer wall of OARs (bladder, rectum, sigmoid) on all the 55 sets of CT images. Rectum was contoured from above the anal sphincter to the level of transition to sigmoid and sigmoid was contoured from the recto-sigmoid flexure. Original treatment plans were assessed for the respective applications with the contours now drawn. DVH parameters for minimum dose to the most irradiated contiguous volume of $0.1 \mathrm{cc}, 1 \mathrm{cc}$ and $2 \mathrm{cc}$ (D0.1, D1cc, and D2cc respectively) were produced for each OARs with 100000 sample points. ICRU point doses for bladder (bICRU) and rectum (rICRU) were recorded. Total dose was computed separately for OARs using ICRU point dose and D2cc. Total dose represents absorbed dose contributed by both EBRT and ICBT with the assumption that the OARs received full dose from EBRT and the same area of OARs were irradiated for all ICBT fractions. The difference in total dose when calculated using the ICRU point dose versus D2cc was analyzed. Physical doses were converted to a biologically equivalent dose and normalized to conventional 2 Gy fractions $(\alpha / \beta=3)$, EQD2.

\section{Data analysis}

All parameters were tested for normal distribution. Paired T-test was used as a parametric test to compare means of ICRU point doses and D2cc volume doses as well as the total dose computed, using these two parameters. Mean ratio was also calculated as an average of each application.

\section{Results}

Table 1 shows the comparison of means between ICRU point doses and D2cc volume doses. Mean ICRU and D2cc doses calculated as an average of each fraction presented a statistically significant difference for bladder $(p=0.000)$ but no difference was found for rectum $(p=0.055)$. Similarly, comparison of mean total dose (ICRU point Vs D2cc) for each patient was found to be significantly different for bladder $(p=0.004)$ but not for rectum $(p=0.090)$. For bladder, bICRU underestimated D2cc dose with an average ratio of $1.34 \pm 0.34$ and total dose computed using bICRU underestimated total dose computed using D2cc with an average ratio of $1.16 \pm 0.12$.

Comparison of values from this study with other published values $[10,11]$ can be found in Table 2 . In general, various dose parameters for bladder from this study were lower than in two others published data, while rectum and sigmoid doses were higher in this study.

3 out of 10 patients, 7 out of 10 patients, and 5 out of 10 patients exceeded the (GYN) GEC-ESTRO Working Group [9] recommended dose constraint for bladder, rectum, and sigmoid, respectively (Table 3).

\section{Discussion}

The whole volume (outer wall) of OARs was contoured in this study. Organ and organ wall contouring yield comparable results when volumes up to $3 \mathrm{cc}$ are considered $[12,13] .0 .1 \mathrm{cc}, 1 \mathrm{cc}$, and $2 \mathrm{cc}$ volumes were being analyzed, focusing on the 2cc parameter. Currently, NCIS uses only CT-based planning for all ICBT applications. CT images were found to be comparable to MRI images for delineation of outer wall of OARs [14]. Interobserver variation was not addressed in this study.

\section{Bladder}

Results from this study showed that bICRU underestimated bladder D2cc dose by a ratio of 1.34. Similar trend 
Table 1. Comparison of means for ICRU point doses and D2cc volume doses in bladder and rectum

\begin{tabular}{|c|c|c|}
\hline $\mathrm{BT}^{1}$ & Bladder & Rectum \\
\hline Mean ICRU ${ }^{2}(G y)$ & 2.9 (range: $1.2-4.5)$ & 3.4 (range: $2.4-4.2$ ) \\
\hline Mean D2cc 3 (Gy) & 3.9 (range: $1.3-6.3$ ) & 3.6 (range: 1.8-5.9) \\
\hline Paired T-Test & $P=0.000,95 \% \mathrm{Cl}(-1.18,-0.71)$ & $P=0.055,95 \% \mathrm{Cl}(-0.41,0.01)$ \\
\hline Average Ratio (D2cc/ICRU) & $1.34 \pm 0.34$ & $1.07 \pm 0.25$ \\
\hline $\mathrm{EBRT}^{4}+\mathrm{BT}$ & Bladder & Rectum \\
\hline Mean Total Dose using ICRU (Gy $\left.\mathrm{EQD2}_{\mathrm{E} 2}\right)$ & 67.3 (range: $58.5-78.1$ ) & 71.7 (range: 62.4-81.5) \\
\hline Mean Total Dose using D2cc $\left(\mathrm{Gy}_{\mathrm{EQD} 2}\right)$ & 78.5 (range: 61.6-106.6) & 74.3 (range: 63.9-80.2) \\
\hline Paired T-Test & $P=0.004,95 \% \mathrm{Cl}(-17.89,-4.52)$ & $P=0.090,95 \% \mathrm{Cl}(-5.74,0.50)$ \\
\hline Average Ratio (D2cc/ICRU) & $1.16 \pm 0.12$ & $1.04 \pm 0.06$ \\
\hline $\begin{array}{l}{ }^{1} B T-\text { Brachytherapy } \\
{ }^{2} I C R U-\text { International Commission on } R a \\
{ }^{3} D 2 C C-\text { Minimum dose to the most irrad } \\
{ }^{4} E B R T \text { - External beam radiotherapy }\end{array}$ & $\begin{array}{l}\text { and Measurements (ICRU defined point } \\
\text { lous volume of } 2 c C\end{array}$ & \\
\hline
\end{tabular}

Table 2. Comparison of total dose for OARs with other findings

\begin{tabular}{|c|c|c|c|}
\hline & \multicolumn{3}{|c|}{ Mean $\pm \operatorname{StDev}^{5}(G y \alpha / \beta=3)$} \\
\hline & $\mathrm{NCIS}^{6}$ & $\begin{array}{c}\text { Koom et al. } \\
{[10]}\end{array}$ & $\begin{array}{c}\text { Kirisits et al. } \\
\text { [11] }\end{array}$ \\
\hline \multicolumn{4}{|l|}{ Bladder } \\
\hline ICRU Point & $67 \pm 7$ & $75 \pm 19$ & $75 \pm 16$ \\
\hline ICRU $1.5^{7}$ & $84 \pm 19$ & - & $100 \pm 25$ \\
\hline ICRU 2.0 & $94 \pm 29$ & - & $112 \pm 34$ \\
\hline $\mathrm{D} 0.1 \mathrm{CC} \mathrm{C}^{8}$ & $104 \pm 29$ & $107 \pm 30$ & $121 \pm 25$ \\
\hline D1cc & $85 \pm 18$ & $90 \pm 18$ & $92 \pm 11$ \\
\hline $\mathrm{D} 2 \mathrm{cc}$ & $79 \pm 15$ & $84 \pm 15$ & $83 \pm 9$ \\
\hline \multicolumn{4}{|l|}{ Rectum } \\
\hline ICRU Point & $72 \pm 6$ & $69 \pm 10$ & $69 \pm 13$ \\
\hline D0.1cc & $95 \pm 0$ & $80 \pm 14$ & $77 \pm 10$ \\
\hline D1cc & $80 \pm 7$ & $71 \pm 10$ & $66 \pm 7$ \\
\hline $\mathrm{D} 2 \mathrm{cc}$ & $74 \pm 6$ & $67 \pm 9$ & $64 \pm 6$ \\
\hline \multicolumn{4}{|l|}{ Sigmoid } \\
\hline D0.1cc & $102 \pm 22$ & $82 \pm 20$ & $79 \pm 12$ \\
\hline D1cc & $82 \pm 12$ & $71 \pm 13$ & $67 \pm 8$ \\
\hline $\mathrm{D} 2 \mathrm{cc}$ & $76 \pm 10$ & $67 \pm 11$ & $63 \pm 7$ \\
\hline \multicolumn{4}{|c|}{$\begin{array}{l}{ }^{5} \text { StDev - Standard deviation } \\
{ }^{6} \mathrm{NCIS}-\text { National University Cancer Institute, Singapore } \\
7 \text { ICRU } 1.5, I C R U 2.0-\text { Points defined } 1.5 \mathrm{~cm} \text { and } 2.0 \mathrm{~cm} \text { respectively, cra- } \\
\text { nially from the ICRU point } \\
{ }^{8} \text { D0.1cc, D1cc-Minimum dose to the most irradiated contiguous vol- } \\
\text { ume of } 0.1 \mathrm{cc} \text { and } 1 \mathrm{cc} \text { respectively }\end{array}$} \\
\hline
\end{tabular}

was reported by other studies [6, 11, 15-17]. There was also a statistically significant difference in total dose to bladder when using bICRU versus D2cc $(p=0.004)$. bICRU did not accurately reflect correct dose to bladder and it was not a reliable parameter to use as a criteria in planning. Retrospective evaluation of correlation by Kirisits et al. [11]
Table 3. Number of patients with OARs D2cc total dose exceeding the recommended tolerance

\begin{tabular}{|c|c|c|c|}
\hline & Bladder & Rectum & Sigmoid \\
\hline $\begin{array}{l}\text { †Number } \\
\text { of patients }(n=10)\end{array}$ & $3 / 10$ & $7 / 10$ & $5 / 10$ \\
\hline $\begin{array}{l}{ }^{\dagger} \text { Data presented as } \\
\text { exceeding the (GYN) } \\
\text { tolerance (Bladder) }\end{array}$ & $\begin{array}{l}\text { er of patie } \\
\text { ESTRO Wc } \\
\text { OD2, Rectu }\end{array}$ & $\begin{array}{l}\text { th OARs D2 } \\
\text { Group [9] } r \\
\text { Sigmoid > }\end{array}$ & $\begin{array}{l}\text { tal dose } \\
\text { mended } \\
\left(G y_{E Q D 2}\right)\end{array}$ \\
\hline
\end{tabular}

found that D2cc overdose were not reflected in the ICRU reference point and patients subsequently develop Grade 4 late side effects.

Overall comparison of bladder dose parameters with Koom et al. [10] and Kirisits et al. [11] presented that both point dose and volume dose parameters were lower in this study. A review of images used in this study showed that inappropriate location of Foley balloon may have caused the bICRU to move out of the high dose region. The Foley balloon was seen to be 'floating' cranially from the bladder trigone in some cases (Fig. 1). Fluid-filled balloon is recommended to fix Foley balloon in an appropriate location [1]. Besides that, it was also noted that Foley balloon was not properly inflated to the $7 \mathrm{cc}$ volume in a few applications (Fig. 2). These may be the reasons for the low bladder point dose observed in this study. As Koom et al. [10] uses the same applicator model as in this study, the lower bladder volume doses can only be attributed to differences in applicator position and planning (difference in dwell position and dwell time activation).

3 out of 10 patients received a calculated bladder dose (using D2cc definition) exceeding the (GYN) GEC-ESTRO Working Group recommendation [9]. However, no bladder overdose was detected when computed using bICRU (Table 4). Therefore, bICRU may not be a reliable parameter for estimating total dose to the bladder.

\section{Rectum}

There was no difference found between rICRU and D2cc doses $(p=0.055)$ in this study. A few other studies also 


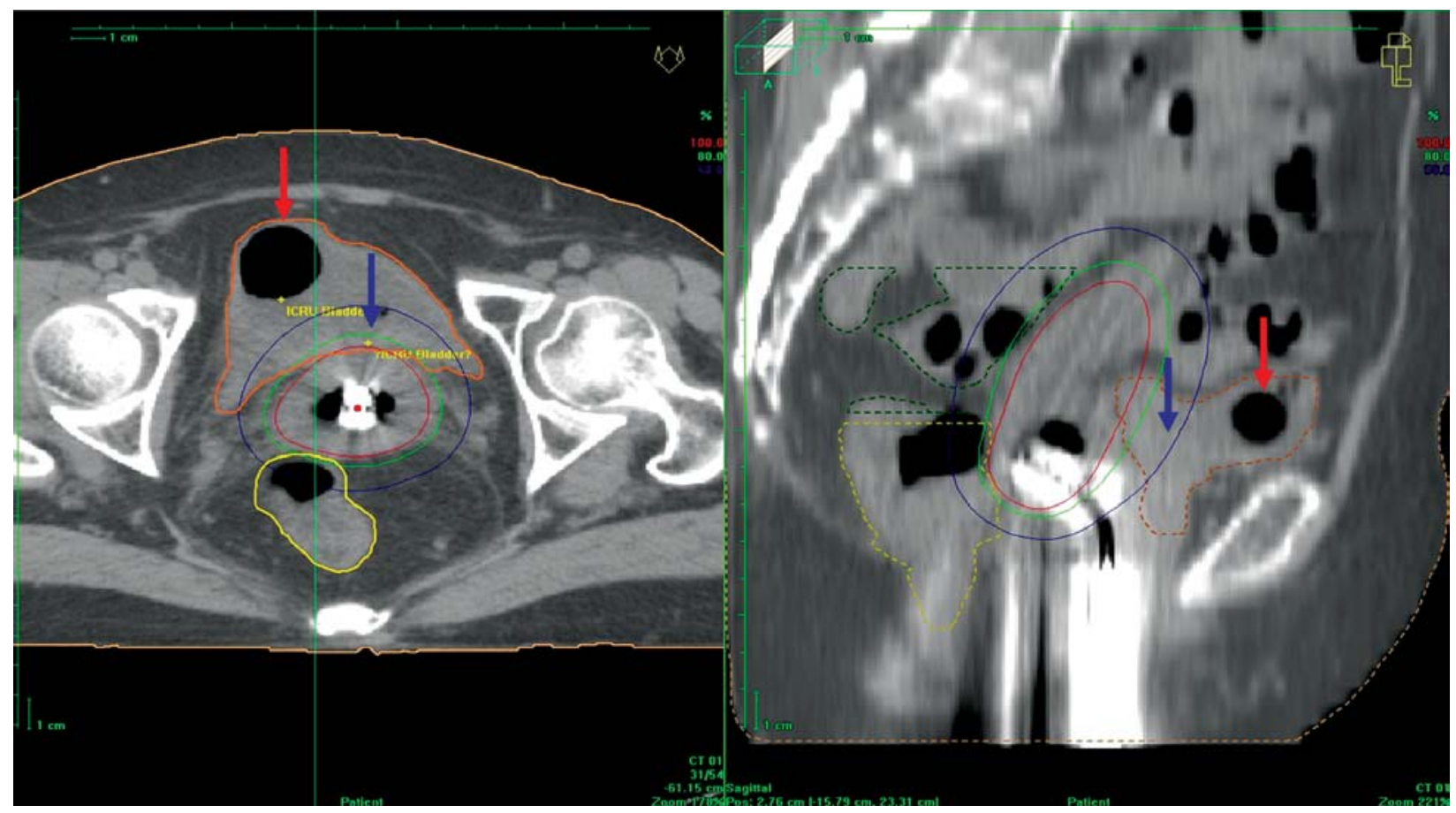

Red Arrow indicates the inappropriate location of Foley balloon during treatment, resulting in inaccurate definition of ICRU bladder point dose. Blue Arrow indicates the appropriate location where the Foley balloon should be.

Fig. 1. Inappropriate placement of Foley balloon

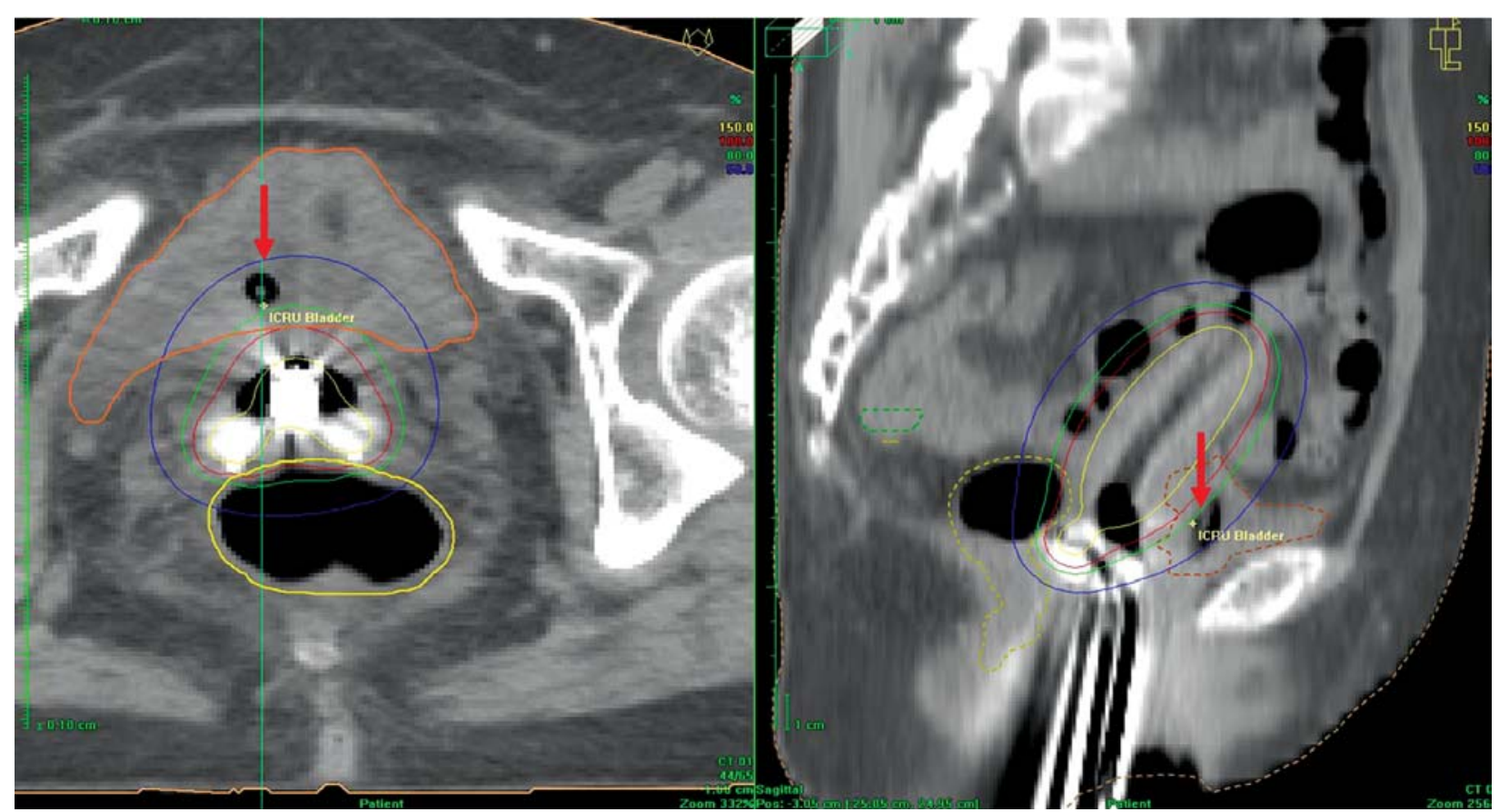

Red Arrow indicates the Foley balloon that was not properly inflated during treatment, resulting in inaccurate definition of ICRU bladder point dose.

Fig. 2. Example of Foley balloon not properly inflated

reported similar finding $[6,11,15,16]$. The average ratio (D2cc/rICRU) in this study was 1.07, similar to the ratio reported by Pelloski et al. [17]. rICRU underestimate D2cc although the difference was not significant $(p=0.055)$. When total dose to rectum was computed for each indi- vidual patient in this study, the difference between rICRU and D2cc was also insignificant $(p=0.090)$. So, it is possible to use rICRU to calculate the total dose to rectum for comparison against the recommended constraint during planning. However, care has to be taken to visually check 
the 3D images (if available) for high dose region especially in the superior part of the rectum. Although ICRU point can represent D2cc in the analysis of total dose to rectum, 7 out of 10 patients exceeded the (GYN) GEC-ESTRO Working Group recommended value [9] because the con-

Table 4. Comparison of bladder total doses computed using bICRU and D2cc for each individual patient

\begin{tabular}{lcc} 
& \multicolumn{2}{c}{ Total Dose $\left(\mathrm{Gy}_{\mathrm{EQD2}}\right)$} \\
\cline { 2 - 3 } & blCRU9 $^{9}$ & Bladder D2cc \\
\hline Pt1 & 59.5 & 66.4 \\
\hline Pt2 & 64.7 & 78.5 \\
\hline Pt3 & 74.0 & 94.8 \\
\hline Pt4 & 78.1 & 92.8 \\
\hline Pt5 & 66.7 & 66.7 \\
\hline Pt6 & 67.5 & 74.7 \\
\hline Pt7 & 64.0 & 75.7 \\
\hline Pt8 & 58.5 & 61.6 \\
\hline Pt9 & 75.9 & 106.6 \\
\hline Pt10 & 64.6 & 67.6 \\
\hline Average & 67.3 & 78.5 \\
\hline StDev & 6.7 & 14.8 \\
\hline Min & 58.5 & 61.6 \\
\hline Max & 78.1 & 106.6
\end{tabular}

9 bICRU - ICRU defined point dose for bladder Bold font: > $90 \mathrm{GY}$ EQD2

Table 5. Comparison of rectum total doses computed using rICRU and D2cc for each individual patient

\begin{tabular}{lcc} 
& \multicolumn{2}{c}{ Total Dose $\left(\mathrm{G}_{\mathrm{EQD} 2}\right)$} \\
\cline { 2 - 3 } & rICRU10 & Rectum D2cc \\
\hline Pt1 & 66.8 & 76.9 \\
\hline Pt2 & $70.9^{*}$ & 76.4 \\
\hline Pt3 & $72.0^{*}$ & 76.6 \\
\hline Pt4 & $79.7^{*}$ & 77.8 \\
\hline Pt5 & $72.2^{*}$ & 78.0 \\
\hline Pt6 & 67.4 & 67.6 \\
\hline Pt7 & 68.9 & 65.2 \\
\hline Pt8 & 62.4 & 63.9 \\
\hline Pt9 & $74.6^{*}$ & 80.2 \\
\hline Pt10 & $81.5^{\star}$ & 80.2 \\
\hline Average & 71.7 & 74.3 \\
\hline StDev & 5.8 & 6.2 \\
\hline Min & 62.4 & 63.9 \\
\hline Max & 81.5 & 80.2
\end{tabular}

10 rICRU - ICRU defined point dose for rectum

Bold font: > $75 G_{E Q D 2}$

* Total dose computed using rICRU point dose can detect overdose if

lower end of the recommended constraint $\left(70 \mathrm{~Gy}_{\mathrm{EQOD}}\right)$ is used. cept of total dose evaluation was not used prospectively during planning in these cases. It was also found that the lower end of the recommended constraint (70 Gy $\left.\mathrm{EQD}_{\mathrm{E} 2}\right)$ should be used if total dose was computed using rICRU dose (Table 5). Bowel preparation is also crucial to avoid
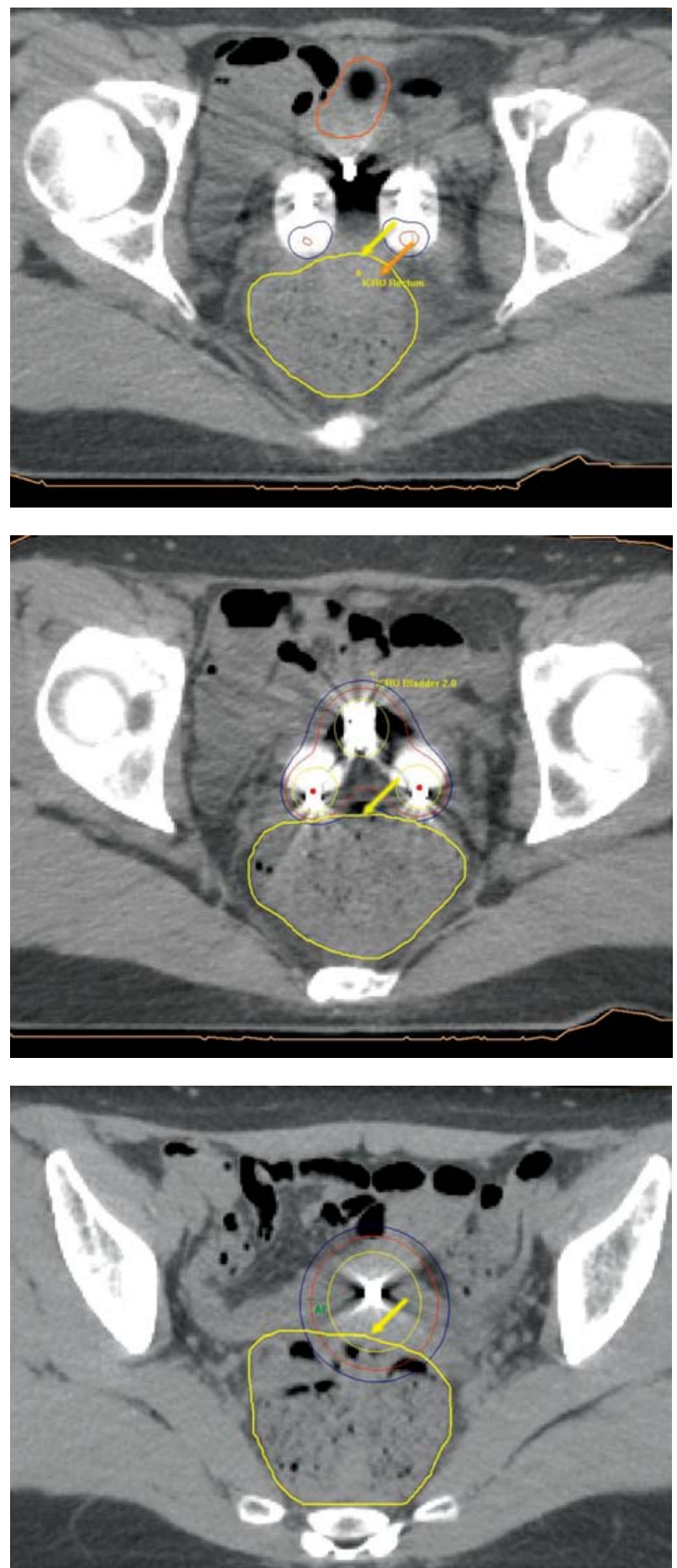

Top to bottom: Consecutive CT images of a patient with full bowel. Yellow Arrow indicates the location of anterior rectal wall. Orange Arrow indicates the rectum point dose defined according to ICRU Report 38.

Fig. 3. Large discrepancies between rICRU dose and D2cc rectum dose in patient with full bowel 
large difference between rICRU dose and D2cc dose that will affect the total dose computation (Fig. 3). Comparison of rectum values with Koom et al. [10] and Kirisits et al. [11] showed that dose to rectum is higher in this study. Review of images used in this work found that poor bowel preparation and inadequate packing may have caused higher volume dose while differences in applicator position and planning may be the reason for the higher ICRU point dose observed. As all procedures were performed under conscious sedation, sufficient packing was difficult. The use of radio-opaque packing in the vagina for better visualization of vaginal and rectal wall as well as careful assessment of ovoid position with respect to the axis of the tandem may help to reduce dose to rectum [6].

\section{Sigmoid}

Dose to sigmoid is traditionally not reported for ICBT. Therefore, no comparison was made to the sigmoid D2cC dose. Although sigmoid dose was not reported in the past because of its mobility, it was noted that sigmoid actually received a substantial amount of dose. Half of the patients evaluated in this study exceeded the dose constraint.

A higher percentage of patient's total dose to rectum (7 out of 10 patients) exceeded the recommended value compared to bladder ( 3 out of 10 patients). This is due to higher tolerance value recommended by (GYN) GECESTRO Working Group [9] for the bladder (80-90 Gy $\left.\mathrm{EQD}_{\mathrm{EQ} 2}\right)$ compared to rectum (70-75 $\mathrm{Gy}_{\mathrm{EQD} 2}$ ) while the ICRU pointbased planning criteria used in our institution is the same for both OARs ( $<80 \%$ of dose to Point A). As a result, more patients received dose exceeding the recommended tolerance value for rectum compared to bladder. The ' $<80 \%$ of dose to Point $\mathrm{A}^{\prime}$ should not be used as generic planning criteria. The percentage should be calculated individually according to the different prescription dose and fractionation using the total dose concept.

\section{Conclusions}

ICRU reference point doses underestimated D2cc volume doses for bladder but no difference were found for rectum. Therefore, rICRU can be used to calculate total dose to rectum but the total dose to bladder should only be computed using the bladder D2cc value. A single generic point dose criterion for both bladder and rectum is not appropriate. Our current institutional practice of point-based planning using the ' $<80 \%$ prescription dose' rule alone resulted in a significant number of patients' OARs doses exceeding the volume tolerance recommended by (GYN) GEC-ESTRO Working Group especially for rectum due to lower dose tolerance. Total dose assessment, even for point-based planning, can reduce the number of overdose incident. ICRU reference point dose should be used with caution for the dose estimation to OARs in ICBT. Volume-based planning should be applied whenever feasible and if not, the total dose concept must be incorporated for all ICBT planning including point-based planning.

\section{References}

1. ICRU. In: Chassagne D, Dutreix A, Almond P et al. ICRU Report No. 38: Dose and volume specification for reporting intracavitary therapy in gynecology. International Commissioning on Radiation Units and Measurements, Bethesda 1985.

2. Pourquier H, Delard R, Achiulle E et al. Quantified approach of the analysis and prevention of urinary complications in radiotherapeutic treatment of cancer of the cervix. Int J Radiat Oncol Biol Phys 1987; 13: 1025-1033.

3. Sinistrero G, Sismondi P, Rumore A et al. Analysis of complications of cervix carcinoma treated by radiotherapy using the Franco-Italian glossary. Int J Radiat Oncol Biol Phys 1993; 26: 203-211.

4. Stryker J A, Bartholomew M, Velkley DE et al. Bladder and rectal complications following radiotherapy for cervix cancer. Gynecol Oncol 1988; 19: 1-11.

5. Katz A, Eifel PJ. Quantification of intracavitary brachytherapy parameters and correlation with outcome in patients with carcinoma of the cervix. Int J Radiat Oncol Biol Phys 2000; 48: 1417-1425.

6. Pelloski CE, Palmer M, Chronowski GM et al. Comparison between CT-based volumetric calculations and ICRU reference point estimates of radiation doses delivered to bladder and rectum during intracavitary radiotherapy for cervical cancer. Int J Radiat Oncol Biol Phys 2005; 62: 131-137.

7. Kim R Y, Shen S, Duan J. Image-based three-dimensional treatment planning of intracavitary brachytherapy for cancer of the cervix: Dose-volume histograms of the bladder, rectum, sigmoid-colon, and small bowel. Brachytherapy 2007; 6: 187-194.

8. Nag S, Erickson B, Thomadsen B et al. The American Brachytherapy Society recommendations for high-dose-rate brachytherapy for carcinoma of the cervix. Int J Radiat Oncol Biol Phys 2000; 48: 201-211.

9. Potter R, Haie-Meder C, Van Limbergen E et al. Recommendations from gynaecological (GYN) GEC ESTRO working group (II): Concepts and terms in 3D image-based treatment planning in cervix cancer brachytherapy - 3D dose volume parameters and aspects of 3D image-based anatomy, radiation physics, radiobiology. Radiother Oncol 2006; 78: 67-77.

10. Koom WS, Sohn DK, Kim JY et al. Computed tomographybased high-dose-rate intracavitary brachytherapy for uterine cervical cancer: Preliminary demonstration of correlation between dose-volume parameters and rectal mucosal changes observed by flexible sigmoidoscopy. Int J Radiat Oncol Biol Phys 2007; 68: 1446-1454.

11. Kirisits C, Potter R, Lang $S$ et al. Dose and volume parameters for MRI-based treatment planning in intracavitary brachytherapy for cervical cancer. Int J Radiat Oncol Biol Phys 2005; 62: 901-911.

12. Wachter-Gerstner N, Wachter S, Reinstadler E et al. Bladder and rectum dose defined from MRI based treatment planning for cervix cancer brachytherapy: Comparison of dose-volume histograms for organ contours and organ wall, comparison with ICRU rectum and bladder reference point. Radiother Oncol 2003; 68: 269-276.

13. Olszewska AM, Saarnak AE, de Boer RW et al. Comparison of dose-volume histograms and dose-wall histograms of the rectum of patients treated with intracavitary brachytherapy. Radiother Oncol 2001; 61: 83-85.

14. Viswanathan A N, Dimopoulos J, Kirisits C et al. Computed tomography versus magnetic resonance imaging-based contouring in cervical cancer brachytherapy: Results of a prospective trial and preliminary guidelines for standardized contours. Int J Radiat Oncol Biol Phys 2007; 68: 491-498.

15. Briot E, de Crevoisier R, Petrow P et al. Dose-volume histogram analysis for tumor and critical organs in intracavitary brachytherapy of cervical cancer with the use of MRI. Radiother Oncol 2001; 60: S3. 
16. Kim RY, Pareek P. Radiography-based treatment planning compared with computed tomography (CT)-based treatment planning for intracavitary brachytherapy in cancer of the cervix: Analysis of dose-volume histograms. Brachytherapy 2003; 2: 200-206

17. Pelloski C R, Palmer M B, Chronowski G M et al. CT based volumetric dosimetry of intracavitary brachytherapy for cervical cancer [Abstract]. Int J Radiat Oncol Biol Phys 2003; 57 (Suppl 1): S343-S344. 